\title{
A Teaching Quality Evaluation Model for Preschool Teachers Based on Deep Learning
}

\author{
https://doi.org/10.3991/ijet.v16i03.20471 \\ Dongjun Ge $\left.{ }^{(}\right)$, Xiaoyue Wang, Jingting Liu \\ Shijiazhuang Preschool Teachers College, Shijiazhuang, China \\ gdj7172@163.com
}

\begin{abstract}
Developed countries regard preschool education as an important starting point and foundation for elite training. In recent years, preschool education has also attracted a growing attention in developing countries like China. Considering the significance of the teaching quality of preschool teachers to lifelong academic achievement, this paper designs a teaching quality evaluation model for preschool teachers based on deep learning. Firstly, a progressive system with a hierarchical structure was developed for the relevant evaluation indices. Then, the fuzzy comprehensive evaluation of each index layer and evaluation criterion was determined by the principle of fuzzy relationship synthesis. Finally, an evaluation prediction model was established based on extreme gradient boosting (XGBoost) algorithm and technology services' ResNet (TS-ResNet), and proved effective and accurate through experiments. The research results provide a reference for the application of the proposed model in other evaluation prediction scenarios.
\end{abstract}

Keywords-Deep learning, extreme gradient boosting (XGBoost), residual network, preschool education, teaching quality evaluation

\section{$1 \quad$ Introduction}

Developed countries generally regard pre-school education as an important starting point and foundation for elite training, therefore they have laid great emphasis on it, and the teaching quality of preschool education and the faculty level of developed countries are improving constantly [1-7]. In China, preschool education is receiving more attention than ever before, and the government has successively issued a few important documents [8-12] such as the Professional Standards for Kindergarten Teachers, the Opinions of the State Council on the Current Development of Preschool Education, and the Guidelines for Kindergarten Education, etc. Although these documents have provided a policy basis for the development of preschool education, in terms of teaching quality assurance system and mode, there is still much room for improvement, and there's an urgent need to build a normative teaching quality evaluation system so as to give objective and scientific evaluation to the teaching quality of preschool teachers. 
As early as the 1990s, the Australian government had proposed that "the teaching quality of preschool teachers has a huge impact on children's lifelong academic achievements, and the key to improving teaching quality is to improve the learning and training quality of preschool teachers" [13]. The 3rd International Summit on the Teaching Profession held in Netherland was themed on the topic of "teaching quality of teachers", the summit gathered scholars and educators from many countries and regions around the global, and the discussed content involved the training schemes, standards, and evaluation of professional preschool teachers [14]. In the National Medium and Long-term Educational Reform and Development Plan Outline, China has also mentioned the need to devote greater efforts to the training of preschool teachers to improve their social status, treatment, and overall qualities [15]. It can be seen that, at home and abroad, the teaching quality of preschool teachers has received wide attention from the society. In terms of the evaluation of teaching quality, scholars have conducted research from multiple aspects and make the evaluation of teaching quality more standardized and professional [16-21]. Burle et al. [22] mentioned that the United States has established quite a few educational evaluation centers and some universities have set up educational evaluation courses. Egon G. TQuba proposed the theory of the fourth generation of educational evaluation based on the concept of value-diversified teaching evaluation, emphasizing that all personnel related to educational evaluation need to participate in the evaluation process [23]. Kassim and Buniyamin [24] analyzed the teaching evaluation results of different subjects and classes of higher education schools and proposed a method to correct the teaching evaluation results based on the differences of classes and subjects, and the feasibility and rationality of the method had been verified with empirical evidence. In order to construct a scientific teaching evaluation index system, Lukianenko and Mischenko [25] analyzed the influencing factors from multiple perspectives including the schools, the teachers and the students, and simplified the index system using the advantage of the RreliefF algorithm in solving nonlinear decision-making problems.

After literature review, we found that most research talked about topics such as the professional standards of preschool teachers or the induction guidance, and there's very few researches concerning the teaching quality assurance system of preschool teachers or the teaching quality evaluation. In the massive teaching evaluation data of preschool teachers, the valuable information has not been mined or scientifically analyzed; and the key influencing factors of the teaching quality of preschool teachers, as well as the correlations among these factors, have not been discovered yet. Based on above concerns, targeting at problems existing in the teaching evaluation process such as numerous evaluation objects, complex evaluation steps, and huge computation load, this paper designs a teaching quality evaluation model for preschool teachers based on deep learning. The main content of the paper is divided into the following parts: the second part constructs a progressive system with a hierarchical structure and applies the principle of fuzzy relationship synthesis to determine the fuzzy comprehensive evaluation of each index layer and evaluation details; the third part establishes the teaching quality evaluation prediction model based on the XGBoost algorithm and the TS-Resnet network model, and the input of the model is the fuzzy comprehensive 
evaluation result of the evaluation object. At last, the effectiveness and accuracy of the constructed model are verified with experiments.

\section{Evaluation Index System for the Teaching Quality of Preschool Teachers}

The prerequisite for constructing an accurate teaching quality evaluation model for preschool teachers is to clarify the hierarchical relationships and interactions among the various evaluation indices. The goal of preschool education is to cultivate socialist builders and successors with comprehensively developed qualities in aspects of morality, intelligence, physique, aesthetics, and labor; therefore, the constructed evaluation index system should take this goal as the starting point, and follow the guidance of documents such as the Professional Standards for Kindergarten Teachers, and the Guidelines for Kindergarten Education. Figure 1 shows the structure of the monitoring system for the teaching quality evaluation of preschool teachers.

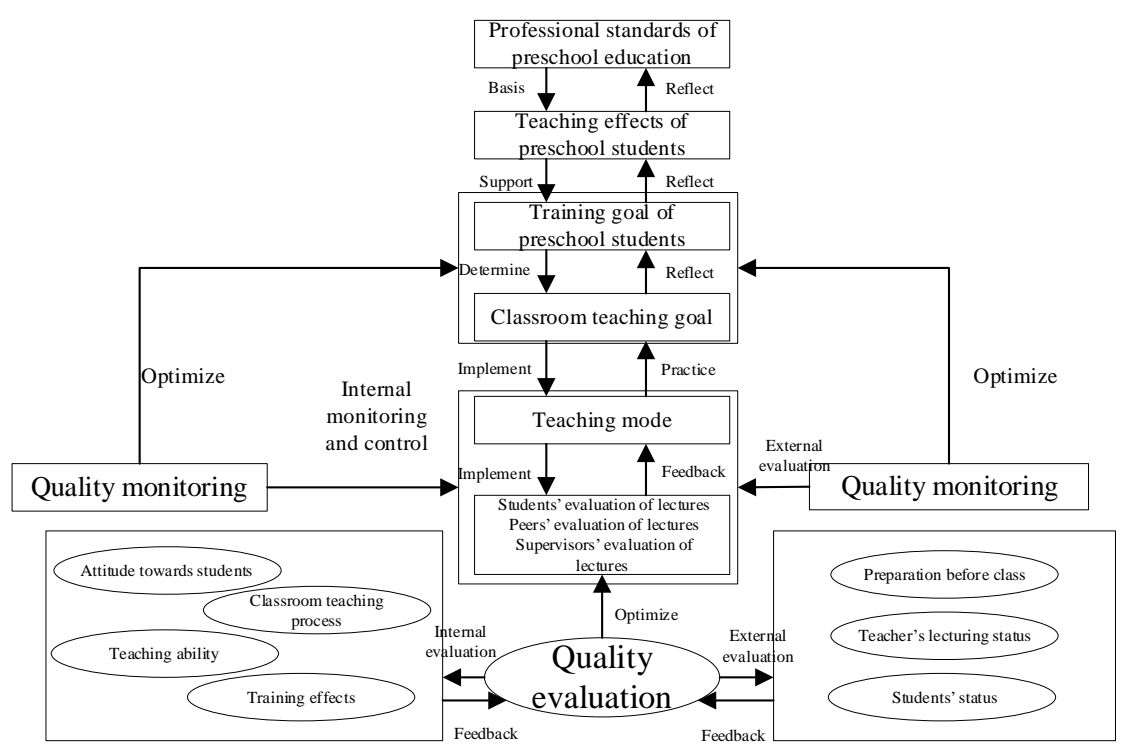

Fig. 1. The monitoring system for the teaching quality evaluation of preschool teachers

When determining the evaluation index system, it is necessary to refer to the traditional teaching quality evaluation methods, and the evaluation objects were divided into three types: preschool students, peer teachers, and supervisor teachers. In this study, the data of evaluation indices were collected via questionnaire survey and interviews, the schools and ages of the respondents (teachers and students) were evenly distributed. A total of 4,500 questionnaires were sent out, 4342 were recovered, the effective rate was $92.8 \%$, and 532 respondents were interviewed. According to the 
survey and interview results, a progressive system with a hierarchical structure was determined for the teaching quality evaluation indices of preschool teachers:

The first layer: object layer

$\mathrm{TQ}=\{\mathrm{TQ} 1, \mathrm{TQ} 2, \mathrm{TQ} 3\}=\{$ students' evaluation of lectures, peers' evaluation of lectures, supervisors' evaluation of lectures \};

The second layer: item layer

TQ1 $=\{$ TQ11, TQ12, TQ13, TQ14 $\}=\{$ attitude towards students; classroom teaching process; teaching ability; training effects $\}$;

TQ2 $=\{$ TQ21, TQ22, TQ23, TQ24 $\}=\{$ teaching attitude; teaching content; teaching method; classroom teaching effects $\}$;

$\mathrm{TQ} 3=\{\mathrm{TQ} 31, \mathrm{TQ} 32, \mathrm{TQ} 33\}=\{$ preparation before class; teacher's teaching status; students' status\};

The third layer: detail layer

TQ11 $=\{$ TQ111, TQ112, TQ113 $\}=\{$ caring for students; treat students patiently and seriously; be amiable and kind to students $\}$;

TQ12 $=\{$ TQ121, TQ122, TQ123 $\}=\{$ clear teaching ideas; key content is highlighted; examples are interesting and appropriate, and can stimulate students' interest $\}$;

$\mathrm{TQ13}=\{\mathrm{TQ131}, \mathrm{TQ132}\}=\{$ novel teaching content; teach students in accordance with their aptitude\};

TQ14=\{TQ141, TQ142, TQ143, TQ144, TQ145 $\}=\{$ students' personality and morals; students' thinking and intelligence; students' physical fitness; students' aesthetics concept; students' civilized and hygiene habits \};

TQ21 $=\{$ TQ211, TQ212, TQ213, TQ214 $\}=\{$ fully prepared lessons; spirited and energetic; familiar with the curriculum content; be enthusiastic and patient with students\};

TQ22 $=\{\mathrm{TQ} 221, \mathrm{TQ} 222, \mathrm{TQ} 223, \mathrm{TQ} 224, \mathrm{TQ} 225\}=\{$ Teaching content is substantial and comprehensive; clear teaching ideas and highlighted key points; appropriate difficulty, amount and teaching speed; novel teaching content; pay attention to the unity of "morality, intelligence, physics, aesthetics and labor" and the integration of theory and practice $\}$;

TQ23= $\{$ TQ231, TQ232, TQ233, TQ234, TQ235, TQ236 $\}=\{$ clear and concise language expression; can well maintain classroom order; diverse and flexible teaching methods; intentionally inspire and train students' brain thinking; good at cultivating students' hands-on ability; handle emergencies wisely\};

$\mathrm{TQ} 24=\{\mathrm{TQ} 241, \mathrm{TQ} 242, \mathrm{TQ} 243\}=\{$ reasonable arrangement of teaching process; fully communicate with students; good teaching atmosphere $\}$;

TQ31 $=\{$ TQ311, TQ312, TQ313, TQ314, TQ315 $\}=\{$ Fully understand the teaching standards and objectives; reasonably make use of existing conditions; reasonably design the teaching content; teaching plans can meet standard requirements; reasonably arrange the teaching progress $\}$;

TQ32 $=\{$ TQ321, TQ322, TQ323, TQ324 $\}=\{$ appropriate difficulty, amount and teaching speed; clear and concise language expression; can maintain classroom order and fully communicate with students; good teaching atmosphere\};

TQ33 $=\{$ TQ331, TQ332, TQ333 $\}=\{$ actively participate in teaching activities; concentration; no violations of discipline $\}$. 
However, the above-mentioned evaluation details are not indices that are easy to quantify; therefore, in order to make the evaluation results of fuzzy indices clearer, this paper applied the principle of fuzzy relationship synthesis to judge the membership degrees of these indices in the detail layer, so as to determine the fuzzy comprehensive evaluation of each index layer and evaluation detail. The specific steps are as follows:

\subsection{Build collections and classify evaluation objects:}

Assume TQpro $=\{$ TQstu, TQft, TQst $\}$ is the evaluation item set of the evaluation objects, Wpro $=\{\mathrm{Wstu}, \mathrm{Wft}, \mathrm{Wst}\}$ is the weight value set of the corresponding evaluation indices. According to the evaluation object, the evaluation indices in the detail layer can be divided into three types:

TQstu $=\{\mathrm{TQstu} 1, \quad \mathrm{TQstu} 2, \ldots, \mathrm{TQstuN1}\} ; \quad \mathrm{TQft}=\{\mathrm{TQft} 1, \quad \mathrm{TQft} 2, \ldots, \mathrm{TQftN} 2\} ;$ TQst $=\{$ TQst1, TQst2,.., TQstN3 $\}$

Above collections meet the following constraints:

1. $\mathrm{N} 1+\mathrm{N} 2+\mathrm{N} 3=\mathrm{NER}$, wherein NER is the total number of evaluation indices in the detail layer; 2) TQstu $\cup$ TQft $\cup$ TQst=TQpro; 3) For any $\forall i, j$ and $i \neq j$, there is $\mathrm{TQi} \cap \mathrm{TQj}=\varnothing$.

The constructed fuzzy comment set of the valuation results is $F C=\{F C 1$, $\mathrm{FC} 2, \ldots, \mathrm{FCM}\}$. The constructed weight sets of the 3 types of evaluation indices in the detail layer are:

$\mathrm{Wstu}=\{\mathrm{W}$ stu1, Wstu2,..,WstuN1 $\} ; \mathrm{Wft}=\{\mathrm{Wft} 1, \mathrm{Wft} 2, \ldots, \mathrm{WftN} 2\} ; \mathrm{Wst}=\{\mathrm{Wst} 1$, Wst $2, \ldots$, WstN3

It can be found that there's one-to-one correspondence relationship between indices and weight values.

2. First-level fuzzy comprehensive evaluation

The first-level single-index evaluation matrix of the three types of evaluation indices in the detail layer is (with TQstu as an example):

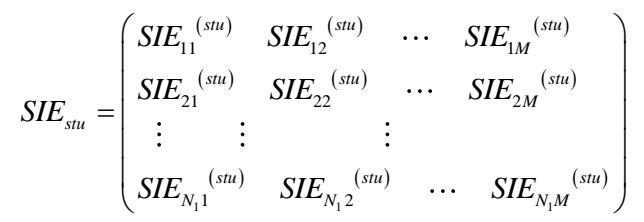

The corresponding first-level fuzzy comprehensive evaluation can be expressed by Formula 2 (with TQstu as an example): 


$$
\begin{aligned}
& F C E_{s t u}=W_{s t u} \cdot S I E_{s t u}
\end{aligned}
$$

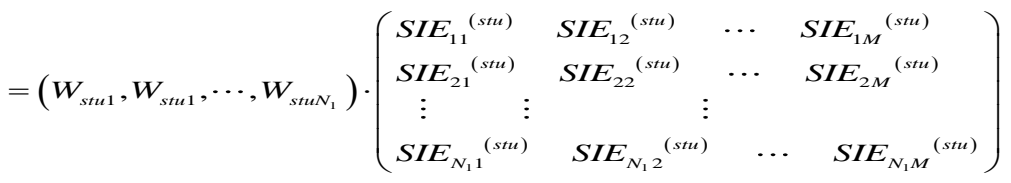

$$
\begin{aligned}
& =\left(F C E_{s t u 1}, F C E_{s t u 2}, \cdots, F C E_{s t u N_{1}}\right)
\end{aligned}
$$

(3) Second-level fuzzy comprehensive evaluation

The first-level fuzzy comprehensive evaluation results constituted the second-level single-index matrix shown as Formula 3:

$$
S I E^{*}=\left(\begin{array}{l}
F C E_{s t u} \\
F C E_{f t} \\
F C E_{s t}
\end{array}\right)
$$

The corresponding second-level fuzzy comprehensive evaluation can be expressed by Formula 4:

$$
\begin{aligned}
& F C E=W_{p r o} \cdot S I E^{*}=\left(W_{s t u}, W_{f t}, W_{s t}\right) \cdot\left(\begin{array}{l}
F C E_{s t u} \\
F C E_{f t} \\
F C E_{s t}
\end{array}\right) \\
& =\left(F C E_{s t u}^{\prime}, F C E_{f t}^{\prime}, F C E_{s t}^{\prime}\right)
\end{aligned}
$$

Figure 2 shows the second-level fuzzy comprehensive evaluation process of the teaching quality of preschool teachers. The evaluation conclusion can be given based on the principle of maximum membership.

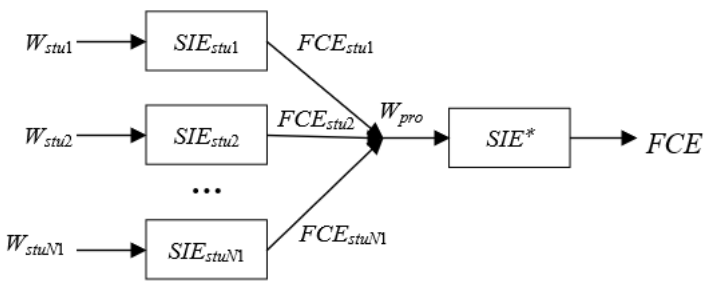

Fig. 2. Process of second-level fuzzy comprehensive evaluation of the teaching quality of preschool teachers

\section{Teaching Quality Evaluation of Preschool Teachers Based on Deep Learning Algorithm and XGBoost Model}

Figure 3 shows the process of the teaching quality evaluation scheme constructed based on deep learning algorithm and XGBoost model. In existing studies that had applied deep learning to teaching quality evaluation, most final classification decisions were given by the full-connection layer of the model. In order to improve the 
accuracy of the evaluation results, this paper used the XGBoost algorithm to replace the full-connection layer in the constructed TS-Resnet network model as the classifier, and the corresponding evaluation model was constructed based on the fuzzy comprehensive evaluation results of the teaching quality of the input evaluation object.

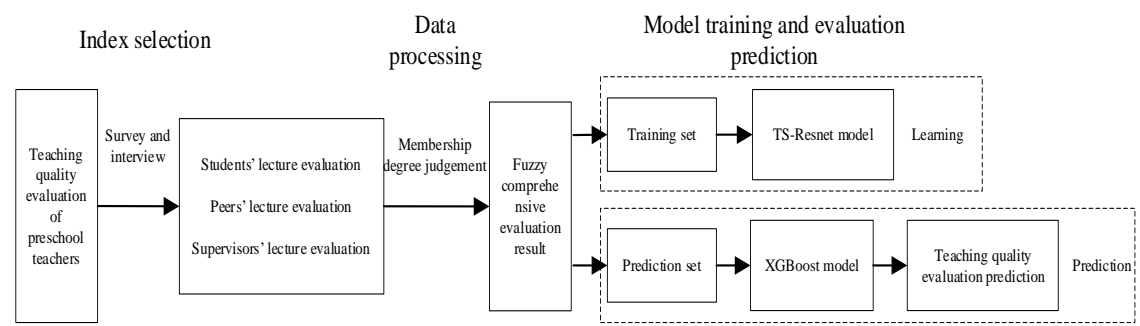

Fig. 3. Process of teaching quality evaluation scheme of preschool teachers

\subsection{TS-Resnet network construction}

Figure 4 shows the teaching quality evaluation model of preschool teachers based on TS-Resnet network. The input data of the TS-Resnet network is a sequence matrix of 44 evaluation detail variables containing fuzzy information of evaluation indices. Assume that the input set I of the model can be expressed by Formula 5 below:

$$
I=\left(I_{1}, I_{2}, \cdots, I_{44}\right)
$$

Assume the number of evaluation samples is $S$, the sequence of the evaluation detail variables has $S$ elements, the i-th evaluation detail variable can be expressed by Formula 6:

$$
I_{i}=\left(I_{i(1)}, I_{i(2)}, \cdots, I_{i(t)}\right)
$$

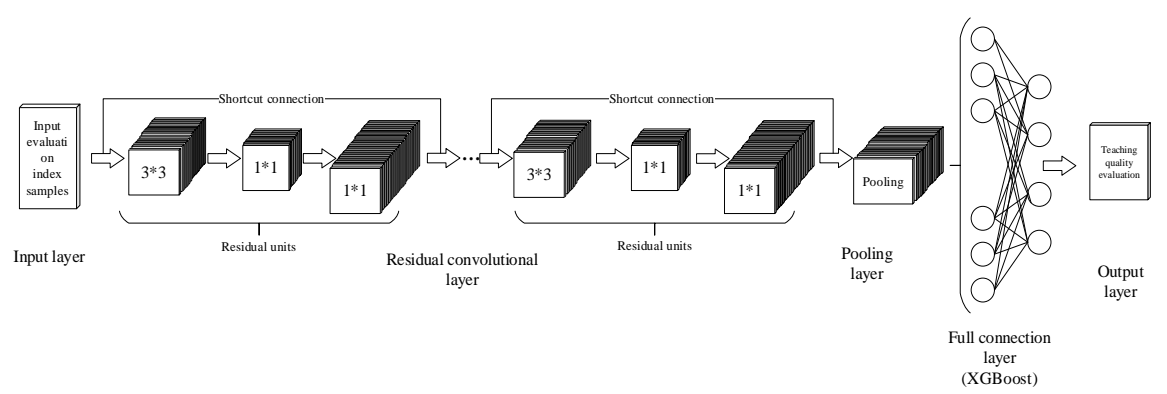

Fig. 4. Teaching quality evaluation model of preschool teachers based on TS-Resnet network

Figure 5 shows the structure of each residual unit in the residual network. It can be seen from the figure that the residual unit processes the data information based on a 
three-layer convolution method which is used for dimensionality reduction, processing, and dimensionality increase. In other words, if the residual network has $\mathrm{R}$ residual units, it contains $3 \mathrm{R}$ convolutional layers, and the input of the $\mathrm{k}$-th convolutional layer can be expressed by Formula 7:

$$
C I_{k}=I_{k-1} \cdot C K_{k}+\varepsilon_{k}
$$

The output can be expressed by Formula 8:

$$
C O_{k}=A F\left(C I_{k}\right)
$$

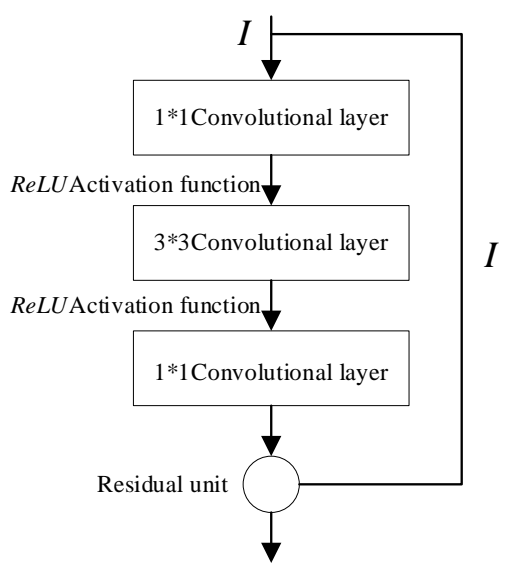

Fig. 5. Residual unit structure

where, $\mathrm{CKk}$ is the convolution kernel of the convolution layer, $\mathrm{kk}$ is the bias coefficient. AF (*) is the activation function. Sigmiod function, Tanh function and ReLU function, the three activation functions that are commonly used in neural networks, are shown as Formulas 9-11:

$$
\begin{gathered}
\operatorname{Sigmiod}(x)=\frac{1}{1+e^{-x}} \\
\operatorname{Tanh}(x)=\frac{e^{x}-e^{-x}}{e^{x}+e^{-x}} \\
\operatorname{ReLU}(x)=\max (0, x)
\end{gathered}
$$

It can be seen that the ReLU function can only activate part of the convolution kernel. When the input is an integer value, the gradient of the function remains at 1 , and when the input is less than 0 , the output remains at 0 . In view of the large differences of index variables among the teaching quality valuation samples of preschool teach- 
ers, after normalization, abnormal evaluation sample index data were excluded by the ReLU function, and the network model can converge quickly.

In Figure 5, the shortcut connection of the residual units can solve the problem that the fitting effect becomes worse as the number of neural network layers increases.

$$
H(I)=F(I)+I
$$

where, I and $\mathrm{H}(\mathrm{I})$ are respectively the input and output matrices of residual unit, $\mathrm{F}(\mathrm{x})$ is the residual. $\mathrm{H}(\mathrm{I})$ is also the input of the pooling layer of the next layer or the input of other residual units. For the neural network with integrated residual units, its layer number is not limited by the worsening of the fitting effect.

For a same evaluation object, the same index variable of multiple evaluation samples has high similarity. In order to reduce computation amount and simplify the neural network, this paper chose to add a mean-pool that meets the data characteristics of the index variables in TS-Resnet so that the model can have a stronger learning ability. The relationship between the input and output of the pooling layer can be expressed by Formula 13:

$$
P O_{l}=\frac{1}{V_{P C}} \sum_{i=1}^{V_{P C}} P I_{l}^{i}
$$

where, VPC is the pooling layer kernel size.

The output layer of the network adopted the weighted summation method shown in Formula 14 to directly calculate the evaluation results of the teaching quality of preschool teachers. The relationship between the input and output of this layer can be expressed by Formula 14:

$$
O=W_{p} O I_{p}+\varepsilon_{p}
$$

where, $\mathrm{Wp}$ is the weight value of the output layer, $\varepsilon \mathrm{p}$ is the bias coefficient of the output layer. The weights and bias coefficients of each layer of the neural network can be obtained through the above-mentioned convolution algorithm, that is, the initialization of the network parameters can be completed. 


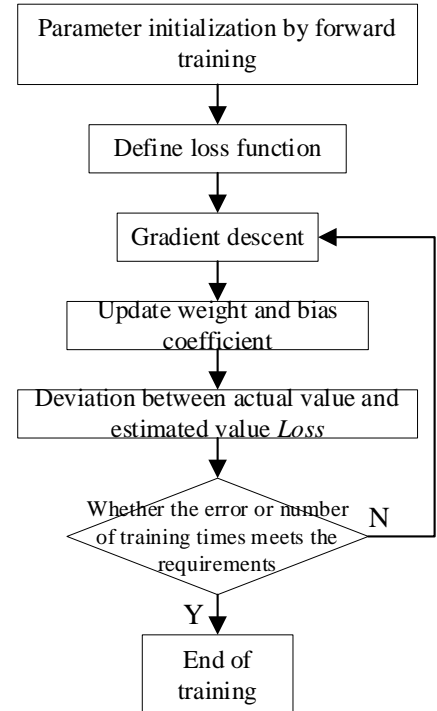

Fig. 6. Flow of the network parameter update algorithm

In order to make the evaluation prediction results more accurate, the model adopted the backward fine-tuning idea of BP neural network to update the network parameters. Figure 6 shows the flow of the update process. The loss function of the model was defined as the mean square error shown in Formula 15:

$$
\text { Loss }=M S E=\frac{1}{S} \sum_{i=1}^{S}\left(E_{a c t}-E_{O}\right)^{2}
$$

where, Eact represents the actual value of the evaluation results of the teaching quality of preschool teachers; EO represents the predicted value of the corresponding evaluation results.

The backward propagation of the network pooling layer to the convolutional layers of each residual unit adopted the gradient descent method. Assume the network has $Q$ convolutional layers, then the sensitivity $\varphi \mathrm{Qq}$ of the q-th convolution kernel of the last convolutional layer can be expressed as:

$$
\phi_{Q}^{q}=\text { upsample }\left(\delta_{Q}^{q+1}\right) \square \operatorname{ReLU}^{\prime}\left(C I_{Q}^{q}\right)
$$

where, upsample(*) is the amplified sensitivity function, and ReLU' (*) is the derivative of the ReLU function. The partial derivative of the loss function to the corresponding convolution kernel bias $\varepsilon Q q$ can be calculated by Formula 17:

$$
P L_{\varepsilon}=\frac{\partial \operatorname{Loss}}{\partial C I_{Q}^{q}} \frac{\partial C I_{Q}^{q}}{\partial \varepsilon_{Q}^{q}}=\sum_{a, b}\left(\phi_{Q}^{q}\right)_{a, b}
$$


where, $a \times b$ is the size of the output of the last convolutional layer. The backpropagation operation process between the pooling layer and the convolutional layer described in the above steps had realized the update of network parameters, and the model had gained higher evaluation prediction accuracy. Unlike the backward propagation described above, the backward propagation between the convolutional layers of the residual units can obtain the propagation gradient by means of the chain rule.

\subsection{XGBoost network construction}

The XGBoost model can more efficiently implement the GBDT algorithm with regression tree as the basic classifier, it has many optimizations and improvements in terms of algorithm and engineering. The core idea of the XGBoost model is to learn a new function by adding trees constantly, and then complete the fitting of the prediction residual of the last evaluation. After the training of D trees is completed, the scores of the evaluation samples need to be predicted. The fuzzy comprehensive evaluation of each sample corresponds to a leaf node on the tree, it has a corresponding evaluation score, and the sum of the scores of each tree is the predicted value of the final evaluation result.

For a given data set $\mathrm{H}=\{(\mathrm{ei}, \mathrm{li})\}$, ei represents the fuzzy comprehensive evaluation set of the teaching quality of preschool teachers, and li is the corresponding fuzzy evaluation label. Suppose hd is the model of the d-th regression tree, then for the model composed of D decision trees, its optimized objective function can be expressed by Formula 18:

$$
\hat{l}_{i}^{(t)}=\sum_{d=1}^{D} f_{d}\left(e_{i}\right)
$$

The above formula can be updated by Formula 19:

$$
\hat{l}_{i}^{(t)}=\hat{l}_{i}^{(t-1)}+f_{D}\left(e_{i}\right)
$$

The loss function of the model can be expressed as Formula 20:

$$
\operatorname{Loss}\left(f_{D}\right)=\sum \operatorname{loss}\left(\hat{l}_{i}^{(t)}, l_{i}^{(t)}\right)+\sum \Gamma\left(f_{D}\right)
$$

where, loss is a differentiable loss function that characterizes the bias between the predicted value and the actual value. $\Omega$ is the regularization term to solve the problem of model overfitting. Assuming Nleaf is the number of leaf nodes of the regression tree, then $\Omega$ can be expressed by Formula 21:

$$
\Omega\left(f_{D}\right)=\gamma N_{\text {leaf }}+\frac{1}{2} \lambda \sum_{j=1}^{N_{\text {leaf }}} \delta_{j}^{2}
$$

where, $\delta$ is the evaluation score corresponding to each leaf node, $\lambda$ and $\gamma$ are parameters that can control the regularization. G1i and G2i are respectively the first- 
order gradient statistics and the second-order gradient statistics, then the loss function can be updated as:

$$
\operatorname{Loss}\left(f_{D}\right) \approx \sum_{j=1}^{N_{\text {leaf }}}\left[\left(\sum_{i \in D} G_{1 i}\right) \delta_{j}+\frac{1}{2}\left(\sum_{i \in D} G_{2 i}+\lambda\right) \delta_{j}^{2}\right]+\gamma N_{\text {leaf }}
$$

In order to improve the evaluation accuracy and speed of the model, before model testing, the hyperparameters of the model need to be updated and optimized. Figure 7 shows the flow of model parameter adjustment and update of TS-Resnet model and XGBoost model.

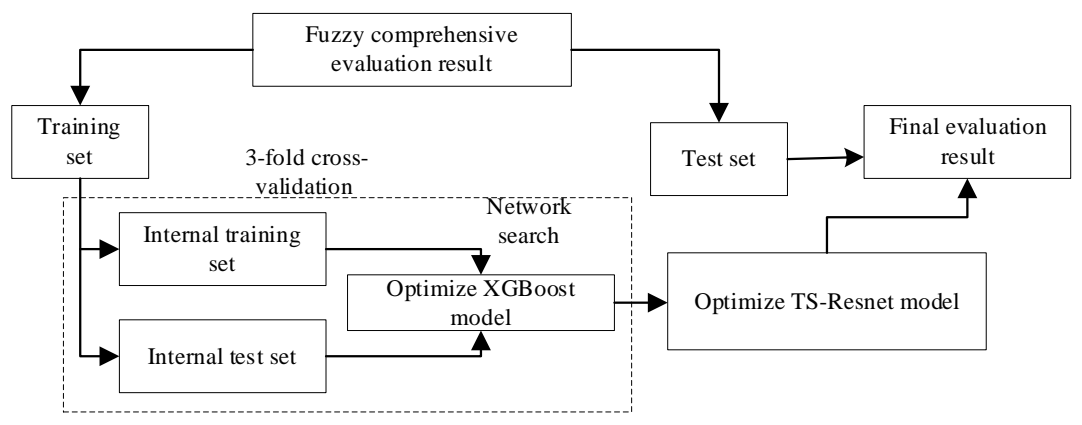

Fig. 7. Flow of model parameter adjustment and update

\section{Experimental Results and Analysis}

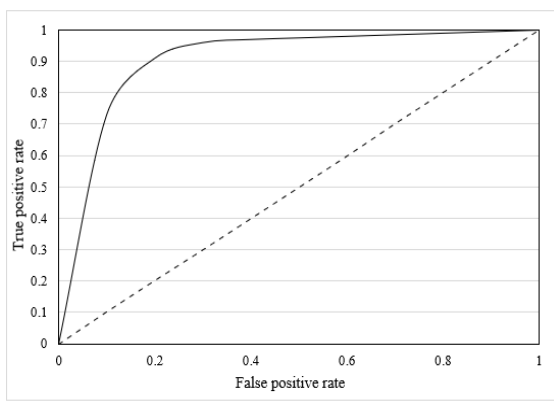

Fig. 8. ROC curve of the algorithm

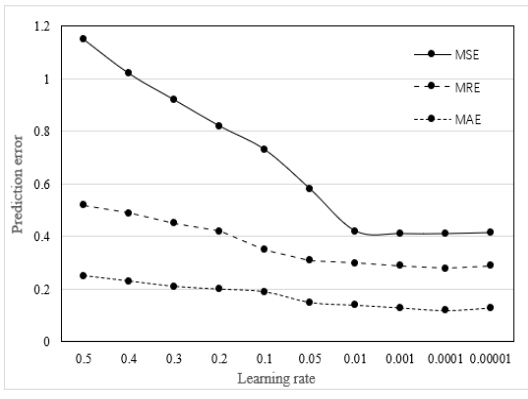

Fig. 9. Evaluation prediction error

The Python language has strong readability, and the TS-Resnet network designed in this paper was built based on the Keras deep learning framework provided by Python. According to the evaluation sample data set of the teaching quality of preschool teachers, this paper constructed training and test data sets for the evaluation model of the teaching quality of preschool teachers. The total number of training samples was 2842 , and the total number of test samples was 1500. Figure 8 shows the ROC (Re- 
ceiver Operating Characteristic) curve of the constructed model. In order to verify whether the constructed neural network evaluation model has the ideal prediction accuracy, this paper designed experiments to compare the network evaluation prediction accuracy and the AUC (Area Under Curve) of ROC curve under different convolution kernel sizes, batch sizes and learning rates.

In this study, the convolution kernel size of the residual network was set to $1 \times 1$, $1 \times 3$ and $3 \times 3$, respectively, and the number of training times was set to 20 rounds. It can be seen from the table that when the size of the convolution kernel of the residual unit was set to $3 \times 3$, the model's performance in evaluating the teaching quality of preschool teachers was the best, the accuracy and AUC of the model reached $91.86 \%$ and $94.01 \%$ respectively. Then, for 4 different batch sizes, this study also designed experiments to compare the evaluation prediction performance of the residual network model. According to the table, when other parameters took fixed values and the batch size took 128, the evaluation accuracy and AUC of the model were the best, respectively $91.97 \%$ and $93.96 \%$. At last, for 4 different learning rates, this study again designed experiments to compare the evaluation prediction performance of the residual network model. According to the table, when the learning rate was set to 0.01 , the model's evaluation prediction performance was the best, and the accuracy and AUC reached $90.46 \%$ and $92.79 \%$.

It can be seen from the above table that the learning rate can affect the evaluation performance of the model. This study designed experiments to compare the evaluation prediction accuracy of the neural network model under different learning rates. Figure 9 shows the experimental results of model evaluation prediction errors under 10 different learning rates. It can be seen from the figure that the three kinds of evaluation prediction errors decreased continuously with the decrease of the learning rate, and they became stable after 0.01 , which once again verified the scientificity of setting the learning rate to 0.01 .

To verify the advantages of the proposed model in evaluating the teaching quality of preschool teachers, this paper took AUC, accuracy, precision, recall, and F1 value as the evaluation indices of the model evaluation prediction performance, and designed comparative experiments. Table 2 shows the prediction results of five models of Naive Bayes, KNN, SVM, decision tree, and random forest. These results were then compared with the results of the proposed model. According to the table, the AUC, accuracy, precision, recall, and F1 values of the proposed model were $93.47 \%$, $91.22 \%, 91.41 \%, 92.89 \%$ and $92.76 \%$, respectively, which were higher than those of the other models, indicating that the proposed residual network model constructed on the evaluation data sets of the teaching quality of preschool teachers had better evaluation prediction effects than the other 5 models.

Preschool teachers need to know their specific scores of each teaching quality evaluation index, then through introspection and self-examination, they can improve their teaching quality, and this is a good cycle. At the same time, by comparing and analyzing the results, education administrators can also understand the teaching level of each teacher through the horizontal comparison of the teaching quality evaluation, and provide reasonable support for management decision-making. Figure 10 shows the horizontal comparison results of teaching quality evaluation of several preschool 
teachers. From the figure, we can know the teaching quality of these preschool teachers from the perspectives of students, peers and supervisors in an intuitive manner.

Table 1. Relationship between neural network parameters and evaluation prediction accuracy

\begin{tabular}{|l|c|c|c|}
\hline \multicolumn{2}{|c|}{ Neural network parameters } & Accuracy & AUC \\
\hline \multirow{3}{*}{ Convolution kernel size } & $1 \times 1$ & $90.87 \%$ & $92.87 \%$ \\
\cline { 2 - 4 } & $1 \times 3$ & $91.24 \%$ & $93.21 \%$ \\
\cline { 2 - 4 } & $3 \times 3$ & $91.86 \%$ & $94.01 \%$ \\
\hline \multirow{3}{*}{ Batch size } & 16 & $90.11 \%$ & $92.32 \%$ \\
\cline { 2 - 4 } & 32 & $90.85 \%$ & $93.10 \%$ \\
\cline { 2 - 4 } & 64 & $91.31 \%$ & $93.42 \%$ \\
\cline { 2 - 4 } & 128 & $91.97 \%$ & $93.96 \%$ \\
\hline \multirow{3}{*}{ Learning rate } & 0.1 & $90.71 \%$ & $92.84 \%$ \\
\cline { 2 - 4 } & 0.01 & $91.32 \%$ & $93.15 \%$ \\
\cline { 2 - 4 } & 0.001 & $90.44 \%$ & $92.48 \%$ \\
\cline { 2 - 4 } & 0.0001 & $90.46 \%$ & $92.79 \%$ \\
\hline
\end{tabular}

Table 2. Evaluation and prediction performance of different models

\begin{tabular}{|l|c|c|c|c|c|}
\hline \multicolumn{1}{|c|}{ Models } & AUC & Accuracy & Precision & Recall & F1-score \\
\hline Naive Bayes Algorithm & $88.25 \%$ & $86.12 \%$ & $88.78 \%$ & $90.13 \%$ & $90.25 \%$ \\
\hline K-Nearest Neighbour algorithm (KNN) & $89.72 \%$ & $88.74 \%$ & $89.98 \%$ & $91.26 \%$ & $90.79 \%$ \\
\hline Support Vector Machines (SVM) & $90.29 \%$ & $89.90 \%$ & $90.16 \%$ & $91.67 \%$ & $91.31 \%$ \\
\hline Decision tree algorithm & $90.71 \%$ & $89.97 \%$ & $90.47 \%$ & $92.04 \%$ & $91.96 \%$ \\
\hline Random forest algorithm & $92.31 \%$ & $90.75 \%$ & $91.21 \%$ & $92.86 \%$ & $92.24 \%$ \\
\hline Proposed model & $93.47 \%$ & $91.22 \%$ & $91.41 \%$ & $92.89 \%$ & $92.76 \%$ \\
\hline
\end{tabular}

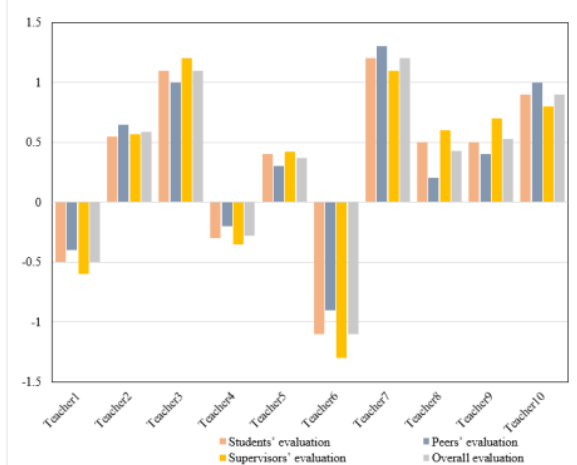

Fig. 10.Horizontal comparison of teaching quality evaluation

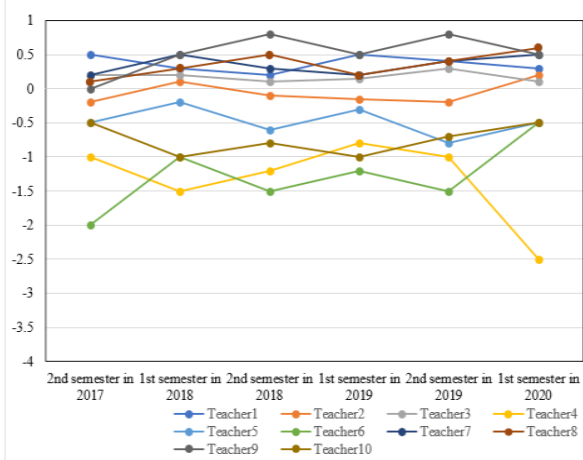

Fig. 11.Longitudinal comparison of teaching quality evaluation

Figure 11 shows the longitudinal comparison results of the teaching quality of preschool teachers in each semester. We can see the changes in the teaching level of each teachers within the period from the 2 nd semester in 2017 to the 1 st semester in 2020. 
Teachers could get the reasons for the changes in the evaluation scores and administrators can understand the stability of the teaching level of teachers and provide reasonable support for decision-making.

\section{Conclusion}

This paper constructed a teaching quality evaluation model for preschool teachers. First, it gave a progressive evaluation index system with a hierarchical structure, and determined the fuzzy comprehensive evaluation of each index layer and evaluation details according to the principle of fuzzy relationship synthesis; then based on the XGBoost algorithm and the TS-Resnet network model, an evaluation prediction model was constructed. The paper constructed the neural network simulation model based on the Keras deep learning framework and designed experiments to compare the evaluation accuracy and AUC of the proposed model under the conditions of different convolution kernel sizes, batch sizes, and learning rates; moreover, the evaluation prediction performance of different models was also compared via experiments, and the experimental results had verified the effectiveness of the model and its advantages in evaluation prediction. This paper provided a reference for the application of the proposed model in other evaluation prediction scenarios.

\section{$6 \quad$ References}

[1] Dhivvya, J.P., Bharath, M., Suresh, A. (2020). Effective teaching using a real-time water quality monitoring prototype. Procedia Computer Science, 172: 43-48. https://doi.org/10. 1016/j.procs.2020.05.006

[2] Sabry, S.S., Sahib, M.A., Nayl, T. (2020). Toward hand functions rehabilitation using the virtual world for pre-school children with cerebral palsy, International Journal of Emerging Technologies in Learning, 15(9): 110-122. https://doi.org/10.3991/ijet.v15i09.13047

[3] Abdi, A.S., Cavus, N. (2019). Developing an electronic device to teach english as a foreign language: Educational toy for pre-kindergarten children, International Journal of Emerging Technologies in Learning, 14(22): 29-44. https://doi.org/10.3991/ijet.v14i22.11747

[4] Nithyanandam, G.K. (2020). A framework to improve the quality of teaching-learning process - A case study. Procedia Computer Science, 172: 92-97. https://doi.org/10.1016/ j.procs.2020.05.013

[5] Perez, B., Castellanos, C., Correal, D. (2020). Measuring the quality of the blended learning approach to teaching computational sciences. Journal of Physics: Conference Series, 1587(1): 19-22. https://doi.org/10.1088/1742-6596/1587/1/012021

[6] Zarqtouni, M. Haddi, A., Allali, H. (2020). Use of formative assessment to improve the online teaching materials content quality. ACM International Conference Proceeding Series, 37: 1-5. https://doi.org/10.1145/3419604.3419808

[7] Espona, M.J., Fisher, C.W. (2015). Teaching information quality to professionals in intelligence government agencies. Proceedings of the 32nd Information Systems Education Conference (ISECON), 2015: 123-132.

[8] Pei, J.Y., Shan, P. (2019). A micro-expression recognition algorithm for students in classroom learning based on convolutional neural network. Traitement du Signal, 36(6): 557-563. https://doi.org/10.18280/ts.360611 
[9] Gold, B., Windscheid, J. (2020). Observing 360-degree classroom videos-Effects of video type on presence, emotions, workload, classroom observations, and ratings of teaching quality. Computers and Education, 156: 103960. https://doi.org/10.1016/j.compedu.2020. $\underline{103960}$

[10] Tjiptady, B.C., Yoto, Marsono. (2020). Entrepreneurship development design based on teaching factory to improve the vocational education quality in singapore and indonesia. 2020 4th International Conference on Vocational Education and Training (ICOVET), 130134. https://doi.org/10.1109/ICOVET50258.2020.9230222

[11] Da Rocha, D.G., Gouveia, L.M.B. (2020). Digital Content Curation for Distance Education: Quality, updating and teaching skills. 2020 15th Iberian Conference on Information Systems and Technologies (CISTI). https://doi.org/10.23919/CISTI49556. 2020.9140942

[12] Tilevich, E., Hall, S., Techapalokul, P. (2020). Teaching the culture of quality from the ground up: Novice-tailored quality improvement for scratch programmers. ASEE Annual Conference and Exposition, Conference Proceedings.

[13] Bouaine, A., Riyami, B., Loukili, M. (2020). Efficient recommendations for improving quality of teaching: Face to face, distance-learning, and learners with disabilities. ACM International Conference Proceeding Series, 20-24. https://doi.org/10.1145/3411681.34116 $\frac{97}{K}$

[14] Kuchkarova, D.F., Achilova, D.A. (2019). Quality management of engineering graphics teaching. Advances in Intelligent Systems and Computing, 809: 1624-1630. https://doi.org/ 10.1007/978-3-319-95588-9_145

[15] Chaki, P.K., Sazal, M.H., Barua, B., Hossain, S., Mohammad, K.S. (2019). An approach of teachers' quality improvement by analyzing teaching evaluations data. 2019 2nd International Conference on Advanced Computational and Communication Paradigms (ICACCP). https://doi.org/10.1109/ICACCP.2019.8882915

[16] Dzedik, V.A., Chigirinskaya, N.V. (2019). Application of Python Software Environment for Analyzing the Systems of Quality Management with the Use of Machine Teaching Methods. Advances in Intelligent Systems and Computing, 726, 573-584. https://doi.org/ 10.1007/978-3-319-90835-9_67

[17] Fourati N., Richard A., Sabouret N., Martin J.C., Chanoni E., Clavel C. (2017). Facial expression of emotions by a virtual narrator for children, Revue d'Intelligence Artificielle, 31(5): 537-556. https://doi.org/10.3166/RIA.31.537-556

[18] Wang, Z. (2020). Recognition and analysis of behavior features of school-age children based on video image processing. Traitement du Signal, 37(4): 603-610. https://doi. org/10.18280/ts. 370408

[19] Müller-Eie, D., Reinertsen, M., Tøssebro, E. (2018). Electronic behaviour mapping and GIS application for stavanger torget, Norway. International Journal of Sustainable Development and Planning, 13(4): 571-581. https://doi.org/10.2495/SDP-V13-N4-571-581

[20] Cesteros, A.M.F.P., Cabezuelo, A. (2016). A proposal to measure the quality of virtual teaching. ACM International Conference Proceeding Series, 155-162. https://doi.org/10.1145/3012430.3012511

[21] McNeil, J.C., Ohland, M.W., Brawner, C.E. (2016). Faculty perspectives and institutional climate for teaching quality in engineering. International Journal of Engineering Education, 32(4): 1801-1812.

[22] Burle, K.J., Ahankari, S.S., Kanai, R.A. (2016). Engineering a better tomorow by bridging the quality gap in education through project-based learning using ICT e-yantra-robot enhanced teaching in engineering colleges. Proceedings-IEEE 8th International Conference on Technology for Education (T4E), 252-253. https://doi.org/10.1109/T4E.2016.065 
[23] Huang, X.Y., Feng, S.Q. (2015). Research on the teaching quality evaluation for the physical education in colleges based on the AHPTOPSIS. Chemical Engineering Transactions, 46: 487-492. https://doi.org/10.3303/CET1546082

[24] Kassim, R.A., Buniyamin, N. (2015). Evaluating teaching quality using data from student online feedback system. 2015 IEEE 7th International Conference on Engineering Education (ICEED), 64-68. https://doi.org/10.1109/ICEED.2015.7451494

[25] Lukianenko, M.S., Mischenko, E.S. (2015). Work in progress: All round questionnaire (ARQ) as the tool for raising quality of teaching. Proceedings of 2015 International Conference on Interactive Collaborative Learning (ICL), 30-33. https://doi.org/10. 1109/ICL.2015.7318169

\section{$7 \quad$ Authors}

Dongjun Ge, she is an associate professor in Shijiazhuang Preschool Teachers College. Her research direction is kindergarten curriculum (email: gdj7172@163.com).

Xiaoyue Wang, she is a lecturer in Shijiazhuang Preschool Teachers College. She received master degree from Southwest University, and her research direction are preschool education and early education (email: 34406082@qq.com).

Jingting Liu, she is an assistant teacher in Shijiazhuang Preschool Teachers College, and received her master degree from University of York, UK. Her research direction is kindergarten curriculum (email: tingting1995528@126.com).

Article submitted 2020-12-17. Resubmitted 2021-01-24. Final acceptance 2021-01-28. Final version published as submitted by the authors. 